\title{
A Catalogue of Three-Level Regular Fractional Factorial Designs
}

\author{
Hongquan Xu \\ Department of Statistics, University of California, Los Angeles, CA 90095-1554, U.S.A. \\ (e-mail: hqxu@stat.ucla.edu)
}

July 25, 2004

\begin{abstract}
A common problem that experimenters face is the choice of fractional factorial designs. Minimum aberration designs are commonly used in practice. There are situations in which other designs meet practical needs better. A catalogue of designs would help experimenters choose the best design. Based on coding theory, new methods are proposed to classify and rank fractional factorial designs efficiently. We have completely enumerated all 27 and 81-run designs, 243-run designs of resolution IV or higher, and 729-run designs of resolution V or higher. A collection of useful fractional factorial designs with 27, 81, 243 and 729 runs is given. This extends the work of Chen, Sun and $\mathrm{Wu}$ (1993), who gave a collection of fractional factorial designs with 16, 27, 32 and 64 runs.
\end{abstract}

Key words: clear effect, linear code, minimum aberration, moment aberration, resolution.

2000 Mathematics Subject Classifications: 62K15, 94B05.

Running title: Three-level fractional factorial designs

\section{Introduction}

Fractional factorial (FF) designs are widely used in various experiments. A common problem experimenters face is the choice of FF designs. An experimenter who has little or no information on the relative sizes of the effects would normally choose a minimum aberration design because it has good overall properties. The minimum aberration criterion (Fries and Hunter, 1980), an extension of the maximum resolution criterion (Box and Hunter, 1961), has been used explicitly or implicitly in the construction of design tables in, among others, National Bureau of Standards (1957), Box, Hunter and Hunter (1978, Table 12.15), Dean and Voss (1999, Tables 15.55 and 15.56), Wu and Hamada (2000, Tables 4A and 5A) and Montgomery (2001, Tables 8-14). The reader is 
referred to $\mathrm{Wu}$ and Hamada (2000) for rich results on minimum aberration designs and extensive references.

An experimenter who has knowledge of the importance of certain main effects and interactions might use a design that guarantees the clear estimation of important effects. For example, in a robust parameter experiment, the experimenter would want to estimate the interactions between control factors and noise factors. There are many cases where minimum aberration designs cannot meet the practical need but other designs can. Different situations call for different designs. A catalogue of designs would help experimenters choose the best design. A collection of FF designs with 16, 27, 32 and 64 runs was given by Chen, Sun and Wu (1993, hereafter CSW).

The main purpose of this paper is to extend the work of CSW for three-level FF designs. We have completely enumerated all 27 and 81-run designs, 243-run designs of resolution IV or higher, and 729-run designs of resolution V or higher. A complete catalogue of 27-run FF designs is given. For 81, 243 and 729 runs, there are too many designs for all to be listed. We carefully choose designs so that the catalogue covers all interesting designs with different properties. Previously, Connor and Zelen (1959) gave a collection of three-level FF designs up to 10 factors and Franklin (1984) gave minimum aberration designs up to 12 factors. A complete catalogue of designs with 27 runs was first given by CSW. Our new catalogue provides more information on the estimation of main effects and interactions. As often done in the literature, the "FF design" in the paper represents only regular fractional factorial designs with resolution at least III.

The extension is not straightforward because the computation is challenging. The original algorithm of CSW failed to construct the complete set of FF designs with 81 runs. We take a coding theory approach and propose new methods to classify and rank designs efficiently. Then we modify their algorithm to construct the catalogue of FF designs with 81, 243 and 729 runs.

In Section 2, we review some basic concepts and definitions for three-level FF designs. We introduce the coding theory approach in Section 3 and the construction method in Section 4. Tables of designs with 27, 81, 243 and 729 runs are given in Section 5 with comments. Concluding remarks are given in Section 6.

\section{Basic concepts and definitions}

We explain some basic concepts briefly through an example; see standard textbooks such as Kempthorne (1952), Dean and Voss (1999), Wu and Hamada (2000) and Montgomery (2001) for 
Table 1: Two designs of 27 runs and 5 factors

\begin{tabular}{|c|c|c|c|c|c|c|c|c|c|c|c|}
\hline Run & $A$ & $B$ & $C$ & $D$ & $E$ & Run & $A$ & $B$ & $C$ & $D$ & $E$ \\
\hline 1 & 0 & 0 & 0 & 0 & 0 & 1 & 0 & 0 & 0 & 0 & 0 \\
\hline 2 & 0 & 0 & 1 & 1 & 0 & 2 & 0 & 0 & 2 & 0 & 0 \\
\hline 3 & 0 & 0 & 2 & 2 & 0 & 3 & 0 & 0 & 1 & 0 & 0 \\
\hline 4 & 0 & 1 & 0 & 1 & 2 & 4 & 0 & 1 & 1 & 2 & 2 \\
\hline 5 & 0 & 1 & 1 & 2 & 2 & 5 & 0 & 1 & 0 & 2 & 2 \\
\hline 6 & 0 & 1 & 2 & 0 & 2 & 6 & 0 & 1 & 2 & 2 & 2 \\
\hline 7 & 0 & 2 & 0 & 2 & 1 & 7 & 0 & 2 & 2 & 1 & 1 \\
\hline 8 & 0 & 2 & 1 & 0 & 1 & 8 & 0 & 2 & 1 & 1 & 1 \\
\hline 9 & 0 & 2 & 2 & 1 & 1 & 9 & 0 & 2 & 0 & 1 & 1 \\
\hline 10 & 1 & 0 & 0 & 1 & 1 & 10 & 1 & 0 & 1 & 2 & 1 \\
\hline 11 & 1 & 0 & 1 & 2 & 1 & 11 & 1 & 0 & 0 & 2 & 1 \\
\hline 12 & 1 & 0 & 2 & 0 & 1 & 12 & 1 & 0 & 2 & 2 & 1 \\
\hline 13 & 1 & 1 & 0 & 2 & 0 & 13 & 1 & 1 & 2 & 1 & 0 \\
\hline 14 & 1 & 1 & 1 & 0 & 0 & 14 & 1 & 1 & 1 & 1 & 0 \\
\hline 15 & 1 & 1 & 2 & 1 & 0 & 15 & 1 & 1 & 0 & 1 & 0 \\
\hline 16 & 1 & 2 & 0 & 0 & 2 & 16 & 1 & 2 & 0 & 0 & 2 \\
\hline 17 & 1 & 2 & 1 & 1 & 2 & 17 & 1 & 2 & 2 & 0 & 2 \\
\hline 18 & 1 & 2 & 2 & 2 & 2 & 18 & 1 & 2 & 1 & 0 & 2 \\
\hline 19 & 2 & 0 & 0 & 2 & 2 & 19 & 2 & 0 & 2 & 1 & 2 \\
\hline 20 & 2 & 0 & 1 & 0 & 2 & 20 & 2 & 0 & 1 & 1 & 2 \\
\hline 21 & 2 & 0 & 2 & 1 & 2 & 21 & 2 & 0 & 0 & 1 & 2 \\
\hline 22 & 2 & 1 & 0 & 0 & 1 & 22 & 2 & 1 & 0 & 0 & 1 \\
\hline 23 & 2 & 1 & 1 & 1 & 1 & 23 & 2 & 1 & 2 & 0 & 1 \\
\hline 24 & 2 & 1 & 2 & 2 & 1 & 24 & 2 & 1 & 1 & 0 & 1 \\
\hline 25 & 2 & 2 & 0 & 1 & 0 & 25 & 2 & 2 & 1 & 2 & 0 \\
\hline 26 & 2 & 2 & 1 & 2 & 0 & 26 & 2 & 2 & 0 & 2 & 0 \\
\hline 27 & 2 & 2 & 2 & 0 & 0 & 27 & 2 & 2 & 2 & 2 & 0 \\
\hline
\end{tabular}


detailed descriptions and more examples.

Table 1 shows two FF designs of 27 runs and five factors, represented as two $27 \times 5$ matrices, where each row corresponds to a run (i.e., treatment combination) and each column to a factor. These are three-level FF designs as each column takes on three different values: 0, 1, 2. Label the five columns as $A, B, C, D$, and $E$ and let $x_{1}, x_{2}, \ldots, x_{5}$ denote the levels of the five columns. The first design (i.e., the left design) is constructed as follows: write down all possible $3^{3}=27$ level combinations for the first three columns and then define the last two columns by

$$
x_{4}=x_{1}+x_{2}+x_{3} \quad(\bmod 3), x_{5}=x_{1}+2 x_{2} \quad(\bmod 3) .
$$

Equivalently, we write $D=A B C$ and $E=A B^{2}$, or $I=A B C D^{2}=A B^{2} E^{2}$, where $I$ is the identity element, and $A B C D^{2}$ and $A B^{2} E^{2}$ are called defining words. From these two defining words, the following defining relations can be obtained

$$
I=A B C D^{2}=A^{2} B^{2} C^{2} D=A B^{2} E^{2}=A^{2} B E=A C^{2} D E=A^{2} C D^{2} E^{2}=B C^{2} D E^{2}=B^{2} C D^{2} E,
$$

For a three-level design, words $W$ and $W^{2}$ (e.g., $A B C D^{2}$ and $A^{2} B^{2} C^{2} D$ ) represent the same contrast. To avoid ambiguity, the convention is to set the first nonzero coefficient to be 1 . Then (1) reduces to

$$
I=A B C D^{2}=A B^{2} E^{2}=A C^{2} D E=B C^{2} D E^{2},
$$

which is called the defining contrast subgroup for the design. This design has one word of length three and three words of length four. The resolution is III because the shortest word has length 3 .

A two-factor interaction (2fi) $A \times B$ has two orthogonal components $A B$ and $A B^{2}$, each representing a contrast of 2 degrees of freedom. A main effect or $2 \mathrm{fi}$ component is called clear (Wu and Chen, 1992; Wu and Hamada, 2000, Section 5.4) if it is not aliased with any other main effects or 2fi components. A 2fi, say $a \times b$, is called clear if both of its components, $a b$ and $a b^{2}$, are clear. One can verify that for the first design in Table 1 , the clear effects are $C, D$ and $C D$.

Now look at the second design in Table 1. The defining contrast subgroup is

$$
I=A B D=A B^{2} E^{2}=A D^{2} E=B D^{2} E^{2} .
$$

All four words have length 3 ; therefore, the resolution is III. It has one clear main effect $(C)$ and four clear 2fi's $(A \times C, B \times C, C \times D$ and $C \times E)$.

An important issue is the choice of designs such as the two designs in Table 1. Both designs have the same resolution III. The minimum aberration criterion (defined next) would choose the 
first design because it has one word of length three while the second design has four words of length three. Indeed, the first design is the minimum aberration design. Therefore, the first design is often recommended especially when the experimenter considers all factors being equally important. On the other hand, if the experimenter knows in advance that one factor and some 2fi's involving that factor is important, then the second design is recommended because it has more clear 2fi's. See CSW for further discussions.

In general, an $s^{n-k} \mathrm{FF}$ design is an $N \times n$ matrix, which has $N=s^{n-k}$ runs, $n$ factors, each at $s$ levels. There are $n-k$ independent columns and other $k$ columns are related to the $n-k$ independent columns through defining words. All defining words and the identity element $I$ together form the defining contrast subgroup. The words $W, W^{2}, \ldots, W^{s-1}$ represent the same contrast and therefore they are viewed as the same. There are $\left(s^{k}-1\right) /(s-1)$ distinct words. Let $A_{j}$ be the number of distinct words of length $j$. The vector $\left(A_{1}, \ldots, A_{n}\right)$ is called the wordlength pattern. The resolution is the shortest wordlength. The minimum aberration criterion (Fries and Hunter, 1980) is to sequentially minimize $A_{j}$ for $j=1, \ldots, n$.

For an $s^{n-k}$ FF design, the defining contrast subgroup has $\left(s^{k}-1\right) /(s-1)$ different words, causing some difficulties in computation when $k$ is large (e.g., $k>10$ ). For example, for a $3^{20-16} \mathrm{FF}$ design, there are 21,523,360 words. It is quite inefficient and sometimes impractical to compute the wordlength pattern and find clear effects via counting all words in the defining contrast subgroup and aliasing sets. In the next section, we propose alternative ways to compute the wordlength pattern and find clear effects based on coding theory.

\section{A coding theory approach}

\subsection{Linear codes}

The connection between FF designs and linear codes was first observed by Bose (1961). For an introduction to coding theory, see MacWilliams and Sloane (1977), van Lint (1999) and Hedayat, Sloane and Stufken (1999, chap. 4).

For a prime power $s$, let $G F(s)$ be the finite field of $s$ elements. An $s^{n-k} \mathrm{FF}$ design $D$ is a linear code of length $n$ and dimension $n-k$ over $G F(s)$, called an $[n, n-k]$ code. The defining contrast subgroup of $D$ corresponds to the dual code $D^{\perp}$, an $[n, k]$ linear code that consists of all row vectors $\left(u_{1}, \ldots, u_{n}\right)$ over $G F(s)$ such that $\sum_{i=1}^{n} u_{i} v_{i}=0$ for all $\left(v_{1}, \ldots, v_{n}\right)$ in $D$. When $s$ is a prime, the dual code is also known as the annihilator (Bailey, 1977). 
The Hamming weight of a vector $\left(u_{1}, \ldots, u_{n}\right)$ is the number of nonzero components $u_{i}$. Let $B_{i}(D)$ and $B_{i}\left(D^{\perp}\right)$ be the number of rows with Hamming weight $i$ in $D$ and $D^{\perp}$, respectively. The vectors $\left(B_{0}(D), B_{1}(D), \ldots, B_{n}(D)\right)$ and $\left(B_{0}\left(D^{\perp}\right), B_{1}\left(D^{\perp}\right), \ldots, B_{n}\left(D^{\perp}\right)\right)$ are called the weight distributions of $D$ and $D^{\perp}$.

The weight distributions of $D$ and $D^{\perp}$ are related through the MacWilliams identities and Pless power moment identities, two fundamental results in coding theory.

Lemma 1. For an $s^{n-k} F F$ design $D$ and $j=0,1, \ldots, n$,

$$
\begin{aligned}
B_{j}\left(D^{\perp}\right) & =s^{-(n-k)} \sum_{i=0}^{n} P_{j}(i ; n, s) B_{i}(D), \\
B_{j}(D) & =s^{-k} \sum_{i=0}^{n} P_{j}(i ; n, s) B_{i}\left(D^{\perp}\right),
\end{aligned}
$$

where $P_{j}(x ; n, s)=\sum_{i=0}^{j}(-1)^{i}(s-1)^{j-i}\left(\begin{array}{c}x \\ i\end{array}\right)\left(\begin{array}{c}n-x \\ j-i\end{array}\right)$ are the Krawtchouk polynomials.

Lemma 2. For an $s^{n-k} F F$ design $D$ and positive integers $t$

$$
\sum_{i=0}^{n} i^{t} B_{i}(D)=s^{n-k} \sum_{i=0}^{\min (n, t)} Q_{t}(i ; n, s) B_{i}\left(D^{\perp}\right),
$$

where $Q_{t}(i ; n, s)=(-1)^{i} \sum_{j=0}^{t} j ! S(t, j) s^{-j}(s-1)^{j-i}\left(\begin{array}{c}n-i \\ j-i\end{array}\right)$ and $S(t, j)=(1 / j !) \sum_{i=0}^{j}(-1)^{j-i}\left(\begin{array}{l}j \\ i\end{array}\right) i^{t} i s$ a Stirling number of the second kind.

The equations (2) and (3) are known as the MacWilliams identities. The equation (4) is known as the Pless power moment identities after Pless (1963).

The wordlength pattern of $D$ is proportional to the weight distribution of the dual $D^{\perp}$ as follows:

$$
A_{i}(D)=B_{i}\left(D^{\perp}\right) /(s-1) \text { for } i=1, \ldots, n,
$$

As a result, the wordlength pattern can be computed through MacWilliams identities (2). In the following we introduce another convenient approach due to $\mathrm{Xu}(2001,2003)$ that uses the Pless power moment identities (4).

\subsection{Minimum moment aberration criterion}

For an $N \times n$ matrix $X=\left(x_{i k}\right)$ and positive integers $t$, define power moments

$$
K_{t}=N^{-2} \sum_{i=1}^{N} \sum_{j=1}^{N}\left(\delta_{i j}\right)^{t}
$$


where $\delta_{i j}$ is the number of coincidences between the $i$ th and $j$ th rows, i.e., the number of $k$ 's such that $x_{i k}=x_{j k}$. For an $s^{n-k} \mathrm{FF}$ design, (5) can be simplified as $K_{t}=N^{-1} \sum_{i=1}^{N}\left(\delta_{i j}\right)^{t}$, where $j$ can be any row number between 1 and $N$. Note that an FF design contains the vector of zeros. Let $C_{i}$ be the number of rows with $i$ zero components. The vector $\left(C_{0}, C_{1}, \ldots, C_{n}\right)$ are called the coincidence distribution. Then (5) becomes

$$
K_{t}=N^{-1} \sum_{i=1}^{n} i^{t} C_{i} .
$$

By applying the Pless power moment identities (4), Xu (2001, 2003) showed that the power moments $K_{t}$ are linear combinations of $A_{1}, \ldots, A_{t}$ as follows.

Theorem 1. For an $s^{n-k} F F$ design and positive integers $t$,

$$
K_{t}=\sum_{i=0}^{t} c_{t}(i ; n, s) A_{i},
$$

where $c_{t}(i ; n, s)=(s-1) \sum_{j=0}^{t}(-1)^{j}\left(\begin{array}{c}t \\ j\end{array}\right) n^{t-j} Q_{j}(i ; n, s)$ for $i=0,1, \ldots, t, Q_{j}(i ; n, s)$ is defined in Lemma $2, A_{0}=1 /(s-1)$ and $A_{i}=0$ when $i>n$. In addition, the leading coefficient of $A_{t}$ in $(7)$ is $c_{t}(t ; n, s)=(s-1) t ! / s^{t}$.

Remark 1. The definition of $K_{t}$ here differs from that in $\mathrm{Xu}(2001,2003)$. Nevertheless, it is evident that they are equivalent up to some constants.

For an $s^{n-k} \mathrm{FF}$ design with resolution at least III, $K_{1}=n / s$ and $K_{2}=n(n+s-1) / s^{2}$ are constants because there are no words of length one or two (i.e., $A_{1}=A_{2}=0$ ). For $s=3$ and $t=3-6,(7)$ becomes

$$
\begin{aligned}
K_{3}= & {\left[12 A_{3}+n\left(2+6 n+n^{2}\right)\right] / 27, } \\
K_{4}= & {\left[48 A_{4}+24(3+2 n) A_{3}+n\left(-6+20 n+12 n^{2}+n^{3}\right)\right] / 81, } \\
K_{5}= & {\left[240 A_{5}+240(2+n) A_{4}+60\left(-3+10 n+2 n^{2}\right) A_{3}+n\left(-30+10 n+80 n^{2}+20 n^{3}+n^{4}\right)\right] / 243, } \\
K_{6}= & {\left[1440 A_{6}+720(5+2 n) A_{5}+720\left(-1+6 n+n^{2}\right) A_{4}+120\left(-39+13 n+21 n^{2}+2 n^{3}\right) A_{3}\right.} \\
& \left.+n\left(42-320 n+270 n^{2}+220 n^{3}+30 n^{4}+n^{5}\right)\right] / 729 .
\end{aligned}
$$

Solving $A_{3}, \ldots, A_{6}$ yields

$$
\begin{aligned}
& A_{3}=\left[27 K_{3}-n\left(2+6 n+n^{2}\right)\right] / 12 \\
& A_{4}=\left[27 K_{4}-18(3+2 n) K_{3}+n\left(6+8 n+6 n^{2}+n^{3}\right)\right] / 16
\end{aligned}
$$




$$
\begin{aligned}
A_{5}= & {\left[81 K_{5}-135(2+n) K_{4}+45\left(15+4 n+2 n^{2}\right) K_{3}\right.} \\
& \left.-n\left(60+110 n+25 n^{2}+10 n^{3}+2 n^{4}\right)\right] / 80 \\
A_{6}= & {\left[729 K_{6}-(3645+1458 n) K_{5}+1215\left(11+3 n+n^{2}\right) K_{4}-135\left(165+80 n+6 n^{2}+4 n^{3}\right) K_{3}\right.} \\
& \left.+n\left(2148+3010 n+1485 n^{2}+175 n^{3}+30 n^{4}+10 n^{5}\right)\right] / 1440
\end{aligned}
$$

Example 1. Consider the first design in Table 1. It is easy to verify that $C_{0}=4, C_{1}=6, C_{2}=14$, $C_{3}=2, C_{4}=0$, and $C_{5}=1$. Definition (6) gives $K_{3}=11, K_{4}=113 / 3, K_{5}=1355 / 9$ and $K_{6}=5995 / 9$. Then equations (8)-(11) yield $A_{3}=1, A_{4}=3, A_{5}=0$ and $A_{6}=0$. Note that equation (11) is valid although $n=5$ here.

Since the power moments $K_{t}$ measure the similarity among runs (i.e., rows), it is natural that a good design should have small power moments. The smaller the $K_{t}$, the better the design. Xu (2001, 2003) proposed the minimum moment aberration criterion which sequentially minimizes $K_{1}, K_{2}, \ldots, K_{n}$.

The following result relates minimum moment aberration and minimum aberration.

Theorem 2. Sequentially minimizing $K_{1}, K_{2}, \ldots, K_{n}$ is equivalent to sequentially minimizing $A_{1}$, $A_{2}, \ldots, A_{n}$. Therefore, designs with less moment aberration have less aberration.

The proof follows from the fact that the leading coefficient of $A_{t}$ in (7) is a positive constant. In this paper we use the minimum moment aberration criterion to rank designs because the power moments are easier to compute than the wordlength patterns.

\subsection{Power moments and clear effects}

Here we introduce a simple method to find clear effects without using the defining contrast subgroup.

To determine whether or not the main effect of column $j$ is clear, for $i=0, \ldots, n-1$, let $\tilde{C}_{i}$ be the number of rows with $i+1$ zero elements and the $j$ th element being zero. Define

$$
\tilde{K}_{2}=\tilde{K}_{2}^{(j)}=N^{-1} \sum_{i=1}^{n-1} i^{2} \tilde{C}_{i}
$$

Theorem 3. For an $s^{n-k} F F$ design,

$$
\tilde{K}_{2}^{(j)} \geq(n-1)(n+s-2) / s^{3}
$$

The main effect of column $j$ is clear if and only if the lower bound is achieved. 
The proof of this and next theorems is beyond the scope of this paper. Interested readers are referred to Xu (2001, Section 4.3), who derived some general identities relating power moments to split wordlength patterns. Theorems 3 and 4 can be verified from these identities.

Example 2. Consider the first design in Table 1. For $n=5$ and $s=3$, the lower bound in (13) is $8 / 9$. First consider column $A$. It is easy to verify that $\tilde{C}_{0}=2, \tilde{C}_{1}=4, \tilde{C}_{2}=2, \tilde{C}_{3}=0$, and $\tilde{C}_{4}=1$. Definition (12) gives $\tilde{K}_{2}=28 / 27$, which is greater than the lower bound; therefore, $A$ is not clear. Next consider column $C$. It is easy to verify that $\tilde{C}_{0}=0, \tilde{C}_{1}=8, \tilde{C}_{2}=0, \tilde{C}_{3}=0$, and $\tilde{C}_{4}=1$. Definition (12) gives $\tilde{K}_{2}=8 / 9$, which is equal to the lower bound; therefore, $C$ is clear.

For any two columns $a$ and $b$, their 2fi componets $a b$ and $a b^{2}$ correspond to column $a+$ column $b \quad(\bmod 3)$ and column $a+2 \times$ column $b \quad(\bmod 3)$. To determine whether or not 2fi component $a b$ is clear, augment column $a b$ to the design matrix. For $i=0, \ldots, n$, let $\hat{C}_{i}$ be the number of rows with $i+1$ zero elements and the element of column $a b$ being zero. Define

$$
\hat{K}_{2}=N^{-1} \sum_{i=1}^{n} i^{2} \hat{C}_{i}
$$

Theorem 4. For an $s^{n-k} F F$ design,

$$
\hat{K}_{2} \geq[2(s-1)+n(n+s-1)] / s^{3} .
$$

The 2fi component ab is clear if and only if the lower bound is achieved.

The same procedure can be used to determine whether or not 2 fi component $a b^{2}$ is clear.

Example 3. Consider the first design in Table 1. First consider whether or not $C D$ (i.e., the third column in the second design) is clear. It is easy to verify that $\hat{C}_{0}=0, \hat{C}_{1}=6, \hat{C}_{2}=2, \hat{C}_{3}=0$, $\hat{C}_{4}=0$, and $\hat{C}_{5}=1$. Definition (14) gives $\hat{K}_{2}=13 / 9$, which is equal to the lower bound in Theorem 4; therefore, $C D$ is clear. Next consider whether or not $C D^{2}$ (i.e., the fourth column in the second design) is clear. It is easy to verify that $\hat{C}_{0}=4, \hat{C}_{1}=0, \hat{C}_{2}=2, \hat{C}_{3}=2, \hat{C}_{4}=0$, and $\hat{C}_{5}=1$. Definition (14) gives $\hat{K}_{2}=17 / 9$, which is greater than the lower bound in Theorem 4; therefore, $C D^{2}$ is not clear.

\section{Construction method}

To obtain the complete catalogue, we take a sequential approach as CSW did. We review CSW's construction method, point out some shortcomings of their method and then introduce our method. 
Table 2: Generator matrix for 27-run designs

\begin{tabular}{cccccccccccccc}
\hline & $\mathbf{1}$ & $\mathbf{2}$ & 3 & 4 & $\mathbf{5}$ & 6 & 7 & 8 & 9 & 10 & 11 & 12 & 13 \\
\hline $\mathrm{a}$ & 1 & 0 & 1 & 1 & 0 & 1 & 0 & 1 & 1 & 1 & 0 & 1 & 1 \\
$\mathrm{~b}$ & 0 & 1 & 1 & 2 & 0 & 0 & 1 & 1 & 2 & 0 & 1 & 1 & 2 \\
$\mathrm{c}$ & 0 & 0 & 0 & 0 & 1 & 1 & 1 & 1 & 1 & 2 & 2 & 2 & 2 \\
\hline
\end{tabular}

\subsection{Basic idea}

Let $r=n-k, N=s^{r}$ and $m=(N-1) /(s-1)$. An $s^{n-(n-r)}$ FF design can be viewed as $n$ columns of an $N \times m$ matrix $H$, where $H$ is a saturated FF design with $N$ runs, $m$ factors and $s$ levels. Let $G$ consist of all nonzero $r$-tuples $\left(u_{1}, \ldots, u_{r}\right)^{T}$ from $G F(s)$ in which the first nonzero $u_{i}$ is 1 . Then $G$ is called the generator matrix and $H$ is formed by taking all linear combinations of the rows of $G$. For example, for $s=3$ and $r=3$, the generator matrix $G$ and design matrix $H$ are given in Tables 2 and 3, respectively.

Two designs are isomorphic if one can be obtained from the other by permuting the rows, the columns and the levels of each column.

Let $D_{n}$ be the set of nonisomorphic designs with $n$ columns. CSW constructed $D_{n+1}$ from $D_{n}$ by adding an additional column. For each design in $D_{n}$, there are $m-n$ ways to add a column to produce a design with $n+1$ columns. Let $\tilde{D}_{n+1}$ be the set of these designs. Obviously, $\left|\tilde{D}_{n+1}\right|=(m-n)\left|D_{n}\right|$. CSW showed that $D_{n+1}$ is a subset of $\tilde{D}_{n+1}$. However, some designs in $\tilde{D}_{n+1}$ are isomorphic and therefore it is necessary to eliminate these redundant designs to construct $D_{n+1}$.

To identify nonisomorphic designs, CSW divided all designs into different categories according to their wordlength patterns and letter patterns. The letter pattern counts the frequency of the letters contained in the words of different lengths (Draper and Mitchell, 1970). Obviously, designs in different categories are not isomorphic. However, designs in the same category are not necessarily isomorphic; see Chen and Lin (1991) for a counter example. For designs in the same category, CSW applied a complete isomorphism check procedure to determine whether or not two designs are isomorphic. The complete isomorphism check considers all possible ways of choosing independent columns and relabeling letters and words.

We observe that the use of wordlength patterns and letter patterns is not efficient in identifying nonisomorphic designs for three-level FF designs. A close examination on the complexity shows that letter pattern check might be more time consuming than complete isomorphism check. Indeed, 
Table 3: Design matrix for 27-run designs

\begin{tabular}{rccccccccccccc}
\hline Run & $\mathbf{1}$ & $\mathbf{2}$ & 3 & 4 & $\mathbf{5}$ & 6 & 7 & 8 & 9 & 10 & 11 & 12 & 13 \\
\hline 1 & 0 & 0 & 0 & 0 & 0 & 0 & 0 & 0 & 0 & 0 & 0 & 0 & 0 \\
2 & 0 & 0 & 0 & 0 & 1 & 1 & 1 & 1 & 1 & 2 & 2 & 2 & 2 \\
3 & 0 & 0 & 0 & 0 & 2 & 2 & 2 & 2 & 2 & 1 & 1 & 1 & 1 \\
4 & 0 & 1 & 1 & 2 & 0 & 0 & 1 & 1 & 2 & 0 & 1 & 1 & 2 \\
5 & 0 & 1 & 1 & 2 & 1 & 1 & 2 & 2 & 0 & 2 & 0 & 0 & 1 \\
6 & 0 & 1 & 1 & 2 & 2 & 2 & 0 & 0 & 1 & 1 & 2 & 2 & 0 \\
7 & 0 & 2 & 2 & 1 & 0 & 0 & 2 & 2 & 1 & 0 & 2 & 2 & 1 \\
8 & 0 & 2 & 2 & 1 & 1 & 1 & 0 & 0 & 2 & 2 & 1 & 1 & 0 \\
9 & 0 & 2 & 2 & 1 & 2 & 2 & 1 & 1 & 0 & 1 & 0 & 0 & 2 \\
10 & 1 & 0 & 1 & 1 & 0 & 1 & 0 & 1 & 1 & 1 & 0 & 1 & 1 \\
11 & 1 & 0 & 1 & 1 & 1 & 2 & 1 & 2 & 2 & 0 & 2 & 0 & 0 \\
12 & 1 & 0 & 1 & 1 & 2 & 0 & 2 & 0 & 0 & 2 & 1 & 2 & 2 \\
13 & 1 & 1 & 2 & 0 & 0 & 1 & 1 & 2 & 0 & 1 & 1 & 2 & 0 \\
14 & 1 & 1 & 2 & 0 & 1 & 2 & 2 & 0 & 1 & 0 & 0 & 1 & 2 \\
15 & 1 & 1 & 2 & 0 & 2 & 0 & 0 & 1 & 2 & 2 & 2 & 0 & 1 \\
16 & 1 & 2 & 0 & 2 & 0 & 1 & 2 & 0 & 2 & 1 & 2 & 0 & 2 \\
17 & 1 & 2 & 0 & 2 & 1 & 2 & 0 & 1 & 0 & 0 & 1 & 2 & 1 \\
18 & 1 & 2 & 0 & 2 & 2 & 0 & 1 & 2 & 1 & 2 & 0 & 1 & 0 \\
19 & 2 & 0 & 2 & 2 & 0 & 2 & 0 & 2 & 2 & 2 & 0 & 2 & 2 \\
20 & 2 & 0 & 2 & 2 & 1 & 0 & 1 & 0 & 0 & 1 & 2 & 1 & 1 \\
21 & 2 & 0 & 2 & 2 & 2 & 1 & 2 & 1 & 1 & 0 & 1 & 0 & 0 \\
22 & 2 & 1 & 0 & 1 & 0 & 2 & 1 & 0 & 1 & 2 & 1 & 0 & 1 \\
23 & 2 & 1 & 0 & 1 & 1 & 0 & 2 & 1 & 2 & 1 & 0 & 2 & 0 \\
24 & 2 & 1 & 0 & 1 & 2 & 1 & 0 & 2 & 0 & 0 & 2 & 1 & 2 \\
25 & 2 & 2 & 1 & 0 & 0 & 2 & 2 & 1 & 0 & 2 & 2 & 1 & 0 \\
26 & 2 & 2 & 1 & 0 & 1 & 0 & 0 & 2 & 1 & 1 & 1 & 0 & 2 \\
27 & 2 & 2 & 1 & 0 & 2 & 1 & 1 & 0 & 2 & 0 & 0 & 2 & 1 \\
\hline
\end{tabular}


for $s^{n-(n-r)}$ designs, the complexity of wordlength pattern and letter pattern check is $O\left(n s^{n-r}\right)$ while the complexity of complete isomorphism check is $O\left(n\left(\begin{array}{l}n \\ r\end{array}\right) r !(s-1)^{r}\right)$. The former is much larger than the latter when $n$ is large (for fixed $s>2$ and $r$ ).

Our algorithm differs from CSW's in the way in which designs are categorized. We divide all designs into different categories according to their coincidence distributions and moment projection patterns (to be defined next). The use of coincidence distributions is equivalent to the use of wordlength patterns in terms of distinguishing designs but is more efficient in terms of computation. The use of moment projection patterns is proven to be more efficient than the use of letter patterns in terms of both distinguishing designs and computation. For designs in the same category, we apply the complete isomorphism check as CSW did.

\subsection{Moment projection patterns}

The idea of moment projection patterns comes from some recent work on the isomorphism check of nonregular designs. It is quite often that nonregular designs have the same (generalized) wordlength pattern but different projection properties. The approach taken here is inspired by Clark and Dean (2001) and Ma, Fang, and Lin (2001), who proposed algorithms for identifying nonisomorphic designs by examining some properties of their projection designs. See also Xu and Deng (2002) for a related procedure.

For an $s^{n-(n-r)}$ FF design, consider its projection designs. For each projection design, we can compute the power moments as in (6) for any $t$. For given $p(1 \leq p \leq n)$, there are $\left(\begin{array}{l}n \\ p\end{array}\right)$ projection designs with $p$ columns. The frequency distribution of $K_{t}$-values of these projection designs is called the $p$-dimensional $K_{t}$-value distribution. It is evident that isomorphic designs have the same $p$-dimenionsonal $K_{t}$-value distribution for all positive integers $t$ and $1 \leq p \leq n$. Whenever two designs have different $p$-dimensional $K_{t}$-value distributions for some $t$ and $p$, these two designs must be nonisomorphic.

In the implementation, we fix $t$ arbitrarily at $t=10$ and let $p$ take on values $n-1, n-2, \ldots, n-q$, where $q$ is a pre-chosen number. The choice of $t$ does not make a difference provided $t>5$ in most cases. The complexity of moment projection pattern check is $O\left(n^{q} s^{2 r}\right)$. Recall that the complexity of complete isomorphism check is $O\left(n\left(\begin{array}{l}n \\ r\end{array}\right) r !(s-1)^{r}\right)$ or $O\left(n^{r+1}\right)$ for fixed $s$ and $r$. Therefore, we should choose $q \leq r$. We find the choice of $q=2$ or 3 works well for $s=3$ and $r=4,5,6$.

As an experimentation, we compared the real computer time on identifying all nonisomorphic $3^{15-11}$ designs from nonisomorphic $3^{14-10}$ designs with different choices of $q$. The algorithm took 
more than 67 hours with $q=0$ and about one hour (62-66 minutes) with $q=1,2,3$ on a $1 \mathrm{GHz}$ Mac Xserve. The numbers clearly indicate that the use of moment projection pattern check speeds up the algorithm significantly. We note that with $q=3$, nonisomorphic designs have different coincidence distributions or moment projection patterns; therefore, the complete isomorphism check could be omitted and the time reduced to 14 minutes. Indeed, with $q=3$, all 81-run designs have different coincidence distributions or moment projection patterns; therefore, the complete isomorphism check can be omitted.

\section{A catalogue of selected designs}

We apply the above construction method to obtain the complete collections of designs with 27 and 81 runs. The number of 243-run and 729-run designs is so large that our algorithm fails to produce all designs. Nevertheless, we have obtained the complete collections of 243-run designs with resolution IV or higher and 729-run designs with resolution V or higher. Once all designs are obtained, we rank the designs according to the minimum moment aberration criterion. If two or more designs are equivalent under the minimum moment aberration criterion, which happens when they have the same coincidence distribution (and wordlength pattern), their rankings are arbitrary. Then we compute part of the wordlength pattern $\left(A_{3}, A_{4}, A_{5}, A_{6}\right)$ according to (8)-(11) and find clear effects according to Section 3.3.

The catalogue shows the ranked design, selected columns, wordlength pattern (WLP), the number of clear main effects $(\mathrm{C} 1)$, the number of clear 2fi's $(\mathrm{C} 2)$, the number of clear 2fi components (CC), clear main effects (CME) and clear 2fi's if any. A $3^{n-k} \mathrm{FF}$ design is labeled as $n$-k.i, where $i$ denotes the rank under the minimum moment aberration criterion. The first design $n$ - $k .1$ is always a minimum aberration (MA) $3^{n-k}$ design. An entry such as $a: b$ under the column of clear 2fi's represents the $a \times b$ interaction.

For 81, 243 and 729 runs, there are too many designs for all to be listed. The concept of admissibility (Sun, Wu and Chen, 1997) is useful in selecting designs of interest. For a given number of criteria, a design $d_{1}$ is called to be inadmissible if there exists another design $d_{2}$ such that $d_{2}$ is better than or equal to $d_{1}$ for all the criteria and strictly better than $d_{1}$ for at least one of the criteria. Otherwise, $d_{1}$ is admissible.

We use $\mathrm{C} 1, \mathrm{C} 2$ and $\mathrm{CC}$ to define the admissibility and compile a list of admissible designs with 81, 243 and 729 runs. When two or more admissible designs have the same C1, C2 and CC, only 
the design with lowest rank is given. In most cases, the first three designs ranked by the minimum moment aberration criterion are also given.

\subsection{Designs of 27 runs}

A 27-run FF design has up to 13 columns and Table 2 shows the generator matrix. The independent columns (in boldface) are 1, 2 and 5.

Table 8 gives the complete collection of 27-run designs. There is only one nonisomorphic design for $n=1,2,11$ and 12; therefore, no designs are given. A complete collection of 27-run designs was previously given by CSW. Our rankings are exactly the same as theirs except that we include two more designs 3-0.2 and 4-1.3. These two designs are degenerate and have only nine distinct runs, indicated by an asterisk in the table. Table 8 provides more information than CSW's table. We include $\mathrm{C} 1, \mathrm{C} 2, \mathrm{CC}$ and the actual clear effects whereas CSW report only C2.

Observe that nonisomorphic designs have different wordlength patterns; therefore, wordlength pattern (indeed $A_{3}$ alone) completely determines a 27-run FF design. Since designs are constructed sequentially, we have the following interesting observation. If we arrange the columns in the following order:

12584126111331012 ,

then the first $n$ columns form the MA $3^{n-(n-3)}$ design for $n=1, \ldots, 13$.

Example 4. Look at $3^{5-2}$ designs. The first design 5-2.1 in Table 8 consists of columns 1, 2, 5, 8, 4 (of the design matrix given in Table 3 ). To find the defining words, label the five columns as $A$, $B, C, D$, and $E$. The generator matrix in Table 2 shows that column 8 is the sum of columns 1 , 2 , and $5 \quad(\bmod 3)$ and column $4=$ column $1+2 \times$ column $2 \quad(\bmod 3)$; therefore, $D=A B C$ and $E=A B^{2}$. According to Table 8, this design has one word of length 3 and three words of length 4 $(\mathrm{WLP}=(1,3,0))$, two clear main effects $(\mathrm{C} 1=2)$, no clear 2fi $(\mathrm{C} 2=0)$ and one clear 2fi component $(\mathrm{CC}=1)$. The two clear main effects are $C$ and $D$, which are given as 5 and 8 under CME. Note that design 5-2.1 is indeed the first design given in Table 1.

\subsection{Designs of 81 runs}

An 81-run FF design has up to 40 columns and Table 4 shows the generator matrix. The independent columns (in boldface) are 1, 2, 5 and 14. We apply the algorithm to obtain the complete collection of designs up to 20 columns. This collection also completely classifies all designs with 
Table 4: Generator matrix for 81-run designs

\begin{tabular}{ccccccccccccccccccccc}
\hline & $\mathbf{1}$ & $\mathbf{2}$ & 3 & 4 & $\mathbf{5}$ & 6 & 7 & 8 & 9 & 10 & 11 & 12 & 13 & $\mathbf{1 4}$ & 15 & 16 & 17 & 18 & 19 & 20 \\
\hline $\mathrm{a}$ & 1 & 0 & 1 & 1 & 0 & 1 & 0 & 1 & 1 & 1 & 0 & 1 & 1 & 0 & 1 & 0 & 1 & 1 & 0 & 1 \\
$\mathrm{~b}$ & 0 & 1 & 1 & 2 & 0 & 0 & 1 & 1 & 2 & 0 & 1 & 1 & 2 & 0 & 0 & 1 & 1 & 2 & 0 & 0 \\
$\mathrm{c}$ & 0 & 0 & 0 & 0 & 1 & 1 & 1 & 1 & 1 & 2 & 2 & 2 & 2 & 0 & 0 & 0 & 0 & 0 & 1 & 1 \\
$\mathrm{~d}$ & 0 & 0 & 0 & 0 & 0 & 0 & 0 & 0 & 0 & 0 & 0 & 0 & 0 & 1 & 1 & 1 & 1 & 1 & 1 & 1 \\
& 21 & 22 & 23 & 24 & 25 & 26 & 27 & 28 & 29 & 30 & 31 & 32 & 33 & 34 & 35 & 36 & 37 & 38 & 39 & 40 \\
\hline $\mathrm{a}$ & 0 & 1 & 1 & 1 & 0 & 1 & 1 & 1 & 0 & 1 & 1 & 0 & 1 & 0 & 1 & 1 & 1 & 0 & 1 & 1 \\
$\mathrm{~b}$ & 1 & 1 & 2 & 0 & 1 & 1 & 2 & 0 & 1 & 1 & 2 & 0 & 0 & 1 & 1 & 2 & 0 & 1 & 1 & 2 \\
$\mathrm{c}$ & 1 & 1 & 1 & 2 & 2 & 2 & 2 & 0 & 0 & 0 & 0 & 1 & 1 & 1 & 1 & 1 & 2 & 2 & 2 & 2 \\
$\mathrm{~d}$ & 1 & 1 & 1 & 1 & 1 & 1 & 1 & 2 & 2 & 2 & 2 & 2 & 2 & 2 & 2 & 2 & 2 & 2 & 2 & 2 \\
\hline
\end{tabular}

Table 5: Number of nonisomorphic 81-run designs

\begin{tabular}{lcccccccccccccccccccc}
\hline$n$ & 1 & 2 & 3 & 4 & 5 & 6 & 7 & 8 & 9 & 10 & 11 & 12 & 13 & 14 & 15 & 16 & 17 & 18 & 19 & 20 \\
\hline \# of designs & 1 & 1 & 2 & 4 & 6 & 12 & 23 & 47 & 94 & 201 & 402 & 807 & 1505 & 2659 & 4304 & 6472 & 8846 & 11127 & 12723 & 13358 \\
\hline
\end{tabular}

more than 20 columns. For example, a set of 21 columns corresponds to a unique set of 19 remaining columns (i.e, complementary design). Therefore, by taking the complement of all designs with 19 columns, we obtain all designs with 21 columns. See Suen, Chen and Wu (1997), Xu and Wu (2001) and $\mathrm{Xu}$ (2003) for characterizing MA designs in terms of their complements.

Table 5 shows the number of nonisomorphic designs for $n=1-20$. Here we treat any 27-run design as a (degenerate) 81-run design; therefore, the number of nonisomorphic designs with $n$ columns, $20<n<40$, is equal to the number of nonisomorphic designs with $40-n$ columns.

Table 9 lists selected 81-run designs for $n=5-20$ columns. It includes all designs with resolution IV or higher. There is only one resolution V design, namely design 5-1.1. Resolution IV designs exist for $n=5-10$ columns. The maximum resolution is III when $n \geq 11$.

In all cases, MA 81-run designs are unique up to isomorphism. From Table 9, we have the following result. For $n=3-11$, the first $n$ columns of

$$
12514229243134393
$$

form an MA design; for $n=12-20$, the first $n$ columns of

$$
125142292431325133761873512381516
$$

form an MA design. For $n=21-37$, MA designs can be determined via the complementary design theory. Previously, MA designs for $n \leq 10$ were given by Franklin (1984) and Wu and Hamada 
Table 6: Number of nonisomorphic 243-run designs with resolution IV or higher

\begin{tabular}{lccccccccccccccc}
\hline$n$ & 6 & 7 & 8 & 9 & 10 & 11 & 12 & 13 & 14 & 15 & 16 & 17 & 18 & 19 & 20 \\
\hline \# of designs & 5 & 8 & 19 & 46 & 137 & 356 & 844 & 1532 & 2020 & 1778 & 1019 & 337 & 90 & 20 & 9 \\
\hline
\end{tabular}

(2000, Table 5A.3). These designs are equivalent to MA designs given here.

It is interesting to note that designs with maximum $\mathrm{C} 2$ (or $\mathrm{CC}$ ) are often different from MA designs. For $n=6-10$, maximum $\mathrm{C} 2$ (or CC) designs have resolution III while MA designs have resolution IV. For $n=10-14$, maximum C2 (or CC) designs have a special structure: Column 14 does not appear in any defining words; therefore, column 14 and any 2fi's involving it are clear. For $n \geq 15$, no design has clear effects (i.e., $\mathrm{C} 1=\mathrm{C} 2=\mathrm{CC}=0$ ).

As Franklin (1984) noted, designs given by National Bureau of Standards (Connor and Zelen, 1959) may not have MA. Connor and Zelen (1959) chose resolution IV designs having maximum CC. From Table 9, we observe that there are two cases where MA designs are different from maximum CC resolution IV designs. They recommended design 7-3.2 (plan 27.7.3 in their notation) and design 8-4.2 (plan 81.8.3). These two designs have more clear 2fi components than the competing MA designs 7-3.1 and 8-4.1.

\subsection{Designs of 243 runs}

A 243-run FF design has up to 121 columns. Let $G=\left(y_{1}, y_{2}, \ldots, y_{121}\right)$ be the generator matrix whose columns are defined as

$$
y_{i}=\left(\begin{array}{c}
x_{i} \\
0
\end{array}\right), y_{i+41}=\left(\begin{array}{c}
x_{i} \\
1
\end{array}\right), y_{i+81}=\left(\begin{array}{c}
x_{i} \\
2
\end{array}\right), \text { for } i=1, \ldots, 40,
$$

and $y_{41}=(0,0,0,0,1)^{T}$, where $x_{i}$ is the $i$ th column of the generator matrix for 81-run designs given in Table 4. The independent columns are 1, 2, 5, 14, and 41.

For 243 runs, resolution IV designs have at most 20 columns. Table 6 shows the number of nonisomorphic designs with resolution IV or higher for $n=6-20$. Note that any 81-run design with resolution IV or higher is a (degenerate) 243-run design.

Table 10 lists the selected 243-run designs with resolution IV or higher for $n=6-20$ columns. Because all main effects are clear for resolution IV designs, C1 and clear main effects are omitted in the table.

The most interesting result is that MA 243-run designs are not unique. There are two MA 
Table 7: Number of nonisomorphic 729-run designs with resolution V or higher

\begin{tabular}{lcccccccc}
\hline$n$ & 7 & 8 & 9 & 10 & 11 & 12 & 13 & 14 \\
\hline \# of designs & 4 & 6 & 11 & 22 & 37 & 38 & 6 & 1 \\
\hline
\end{tabular}

designs for $n=14,16,19$ and 20; nine MA designs for $n=17$; and five MA designs for $n=18$. For $n \leq 13$ or $n=15$, MA designs are unique.

For $n \leq 11$, MA designs have resolution V or VI; therefore, no resolution IV designs is given. For $n=7-11$, resolution $\mathrm{V}$ designs are unique. The MA $3^{11-6}$ design 11-6.1 is saturated for a model with all main effects and all 2fi's. Any 7-11 columns of this design form an MA design. For $n=12-15$, MA designs do not have maximum C2; for $n=12-18$, MA designs do not have maximum CC.

Previously, Connor and Zelen (1959) gave designs for $n=6-10$ and Franklin (1984) gave MA designs for $n=7-11$. All these designs are isomorphic to MA designs given here.

\subsection{Designs of 729 runs}

A 729-run FF design has up to 364 columns. Let $G=\left(z_{1}, z_{2}, \ldots, z_{364}\right)$ be the generator matrix whose columns are defined as

$$
z_{i}=\left(\begin{array}{c}
y_{i} \\
0
\end{array}\right), z_{i+122}=\left(\begin{array}{c}
y_{i} \\
1
\end{array}\right), z_{i+243}=\left(\begin{array}{c}
y_{i} \\
2
\end{array}\right), \text { for } i=1, \ldots, 121,
$$

and $z_{122}=(0,0,0,0,0,1)^{T}$, where $y_{i}$ is the $i$ th column of the generator matrix for 243-run FF designs given in Section 5.3. The independent columns are 1, 2, 5, 14, 41, and 122.

For 729 runs, resolution $\mathrm{V}$ designs have at most 14 columns. Table 7 shows the number of nonisomorphic designs with resolution $\mathrm{V}$ or higher for $n=7-14$. Again, any 243-run design with resolution $\mathrm{V}$ or higher is a (degenerate) 729-run design.

Table 11 lists the selected 729-run designs with resolution $\mathrm{V}$ or higher for $n=7-14$ columns. Because all main effects and 2fi's are clear for resolution V designs, C1, C2, CC and clear effects are omitted in the table.

For $n=7-14$, MA designs are unique. For $n=8-12$, there is one unique resolution VI design, i.e., the MA design. Previously, Connor and Zelen (1959) gave designs for $n=7-9$, and Franklin (1984) gave MA designs for $n=8-12$. All these designs are isomorphic to MA designs given here except for one case. For $n=8$, the design given in Connor and Zelen (1959) is isomorphic to design 8-2.2 which has resolution $\mathrm{V}$ while the MA design 8-2.1 has resolution VI. 


\section{Concluding remarks}

Based on coding theory, we use minimum moment aberration and moment projection pattern to classify and rank FF designs, and use power moments to compute wordlength patterns and find clear effects. By modifying CSW's algorithm, we obtain complete collections of 3-level FF designs with 27 and 81 runs, 243 runs with resolution IV or higher and 729 runs with resolution V or higher. Selected designs of interest are given. For easy reference, the complete catalogue is available at the author's web site (http://www. stat.ucla.edu/ ${ }^{\sim} \mathrm{hqxu} / \mathrm{pub} / \mathrm{ffd} 3 /$ ). The online catalogue includes the actual clear 2fi components $a b$ and $a b^{2}$.

One interesting result is that 243-run MA designs are not unique. This is the smallest case known so far where MA designs are not unique. Chen (1992) showed that MA $2^{n-k}$ designs are unique for $k=1,2,3,4$. The catalogue of CSW shows that MA designs are unique for 16, 32 and 64 runs. One interesting question is whether 2-level MA designs are unique. The answer is negative. Bouyukliev and Jaffe (2001) showed that there are exactly seven $[43,7,20]$ linear codes (that is, seven $2^{43-7}$ designs with resolution 20 or higher). According to their complete enumeration, MA

$2^{43-7}$ designs have wordlength pattern $A_{20}=84, A_{24}=35, A_{28}=7, A_{36}=1$ and other $A_{i}=0$; and there are two nonisomorphic designs having this wordlength pattern.

\section{Acknowledgments}

This research was supported by NSF Grant DMS-0204009. The author thanks Dr. Mingyao Ai for pointing out an error in finding clear effects in an early version of the paper. The author also thanks the guest editor and the referees for their valuable comments.

\section{References}

1. Bailey RA (1977) Patterns of confounding in factorial designs. Biometrika 64:597-603

2. Bose RC (1961) On some connections between the design of experiments and information theory. Bull Internat Statist Inst 38:257-271

3. Bouyukliev I, Jaffe DB (2001) Optimal binary linear codes of dimension at most seven. Discrete Math 226:51-70 
4. Box GEP, Hunter JS (1961) The $2^{k-p}$ fractional factorial designs. Technometrics 3:311-351, $449-458$

5. Box GEP, Hunter WG, Hunter JS (1978) Statistics for experimenters. Wiley, New York

6. Chen J (1992) Some results on $2^{n-k}$ fractional factorial designs and search for minimum aberration designs. Ann Statist 20:2124-2141

7. Chen J, Lin DKJ (1991) On the identity relationship of $2^{k-p}$ designs. J Statist Plann Inference 28:95-98

8. Chen J, Sun DX, Wu CFJ (1993) A catalogue of two-level and three-level fractional factorial designs with small runs. Internat Statist Rev 61:131-145

9. Clark JB, Dean AM (2001) Equivalence of fractional factorial designs. Statist Sinica 11:537547

10. Connor WS, Zelen M (1959) Fractional factorial experiment designs for factors at three levels. National Bureau of Standards Applied Mathematics Series 54. US Government Printing Office, Washington, DC

11. Dean AM, Voss DT (1999) Design and analysis of experiments. Springer, New York

12. Draper NR, Mitchell TJ (1970) Construction of a set of 512-run designs of resolution $\geq 5$ and a set of even 1024-run designs of resolution $\geq 6$. Ann Math Statist 41:876-887

13. Franklin MF (1984) Constructing tables of minimum aberration $p^{n-m}$ designs. Technometrics $26: 225-232$

14. Fries A, Hunter WG (1980) Minimum aberration $2^{k-p}$ designs. Technometrics 22:601-608

15. Hedayat AS, Sloane NJA, Stufken J (1999) Orthogonal arrays: theory and applications. Springer, New York

16. Kempthorne O (1952) The design and analysis of experiments. Wiley, New York

17. Ma CX, Fang KT, Lin DKJ (2001) On the isomorphism of fractional factorial designs. J Complexity 17:86-97

18. MacWilliams FJ, Sloane NJA (1977) The theory of error-correcting codes. North-Holland, Amsterdam 
19. Montgomery DC (2001) Design and analysis of experiments. 5th ed. Wiley, New York

20. National Bureau of Standards (1957) Fractional factorial experiment designs for factors at two levels. Applied Mathematics Series 48. US Government Printing Office, Washington DC

21. Pless V (1963) Power moment identities on weight distributions in error correcting codes. Information Control 6:147-152

22. Suen C, Chen H, Wu CFJ (1997) Some identities on $q^{n-m}$ designs with application to minimum aberration designs. Ann Statist 25:1176-1188

23. Sun DX, Wu CFJ, Chen Y (1997) Optimal blocking schemes for $2^{n}$ and $2^{n-p}$ designs. Technometrics 39:298-307

24. van Lint JH (1999) Introduction to coding theory. 3rd ed. Springer, New York

25. Wu CFJ, Chen Y (1992) A graph-aided method for planning two-level experiments when certain interactions are important. Technometrics 34:162-175

26. Wu CFJ, Hamada M (2000) Experiments: planning, analysis and parameter design optimization. Wiley, New York

27. Xu H (2001) Optimal factor assignment for asymmetrical fractional factorial designs: theory and applications. Ph.D. thesis, University of Michigan

28. Xu H (2003) Minimum moment aberration for nonregular designs and supersaturated designs. Statist Sinica 13:691-708

29. Xu H, Deng LY (2002) Moment aberration projection for nonregular fractional factorial designs. UCLA Statistics Electronic Publications, preprint 326 (http://preprints.stat.ucla.edu/)

30. Xu H, Wu CFJ (2001) Generalized minimum aberration for asymmetrical fractional factorial designs. Ann Statist 29:1066-1077 
Table 8: Complete catalogue of 27-run designs

\begin{tabular}{|c|c|c|c|c|c|c|c|}
\hline Design & Columns & WLP & C1 & $\mathrm{C} 2$ & $\mathrm{CC}$ & CME & Clear 2fi's \\
\hline $3-0.1$ & 125 & 0 & 3 & 3 & 6 & all & all \\
\hline $3-0.2^{*}$ & 123 & 1 & 0 & 0 & 0 & & \\
\hline $4-1.1$ & 1258 & 01 & 4 & 0 & 6 & all & \\
\hline $4-1.2$ & 1253 & 10 & 1 & 3 & 6 & 5 & $1: 5 \quad 2: 5 \quad 5: 3$ \\
\hline $4-1.3^{*}$ & 1234 & 40 & 0 & 0 & 0 & & \\
\hline $5-2.1$ & 12584 & 130 & 2 & 0 & 1 & 58 & \\
\hline $5-2.2$ & 12583 & 211 & 0 & 0 & 4 & & \\
\hline $5-2.3$ & 12534 & 400 & 1 & 4 & 8 & 5 & $1: 52: 5 \quad 5: 3 \quad 5: 4$ \\
\hline $6-3.1$ & 1258412 & 2902 & 0 & 0 & 0 & & \\
\hline $6-3.2$ & 125846 & 3631 & 0 & 0 & 0 & & \\
\hline $6-3.3$ & 125836 & 4360 & 0 & 0 & 0 & & \\
\hline $6-3.4$ & 125843 & 5332 & 0 & 0 & 0 & & \\
\hline $7-4.1$ & 12584126 & 51598 & 0 & 0 & 0 & & \\
\hline $7-4.2$ & 1258467 & 611154 & 0 & 0 & 0 & & \\
\hline $7-4.3$ & 1258463 & 710129 & 0 & 0 & 0 & & \\
\hline $7-4.4$ & 12584123 & 89914 & 0 & 0 & 0 & & \\
\hline $8-5.1$ & 1258412611 & 8302432 & 0 & 0 & 0 & & \\
\hline $8-5.2$ & 125841267 & 10233230 & 0 & 0 & 0 & & \\
\hline $8-5.3$ & 125841263 & 11213038 & 0 & 0 & 0 & & \\
\hline $9-6.1$ & 125841261113 & 12545496 & 0 & 0 & 0 & & \\
\hline $9-6.2$ & 12584126113 & 15426996 & 0 & 0 & 0 & & \\
\hline $9-6.3$ & 1258412673 & 163969106 & 0 & 0 & 0 & & \\
\hline $10-7.1$ & 1258412611133 & 2172135240 & 0 & 0 & 0 & & \\
\hline $10-7.2$ & 125841261137 & 2268138250 & 0 & 0 & 0 & & \\
\hline
\end{tabular}

Note: Designs with $n=1,2,11$ or 12 are unique and not listed. An asterisk $(*)$ indicates a degenerate design. 


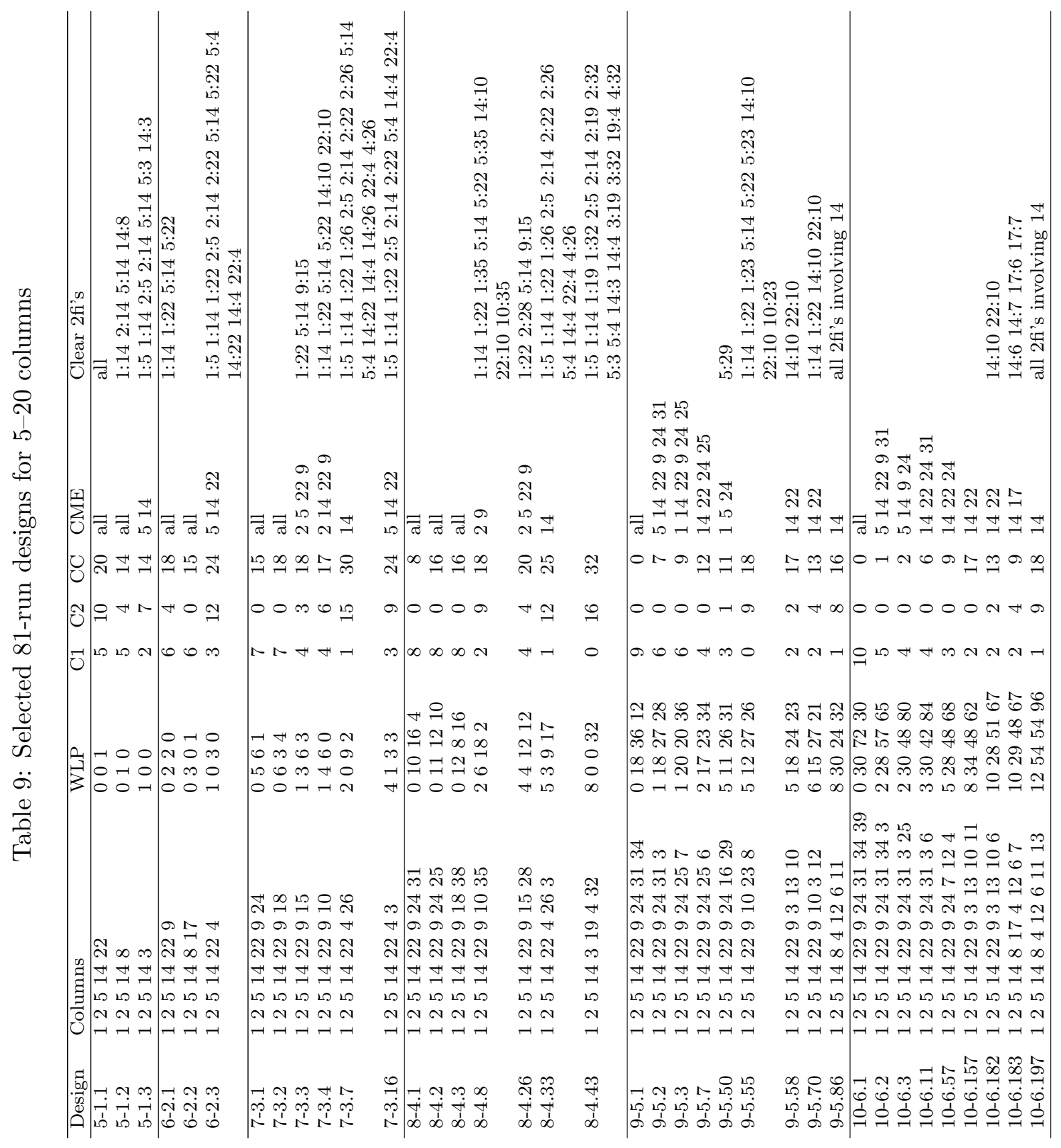




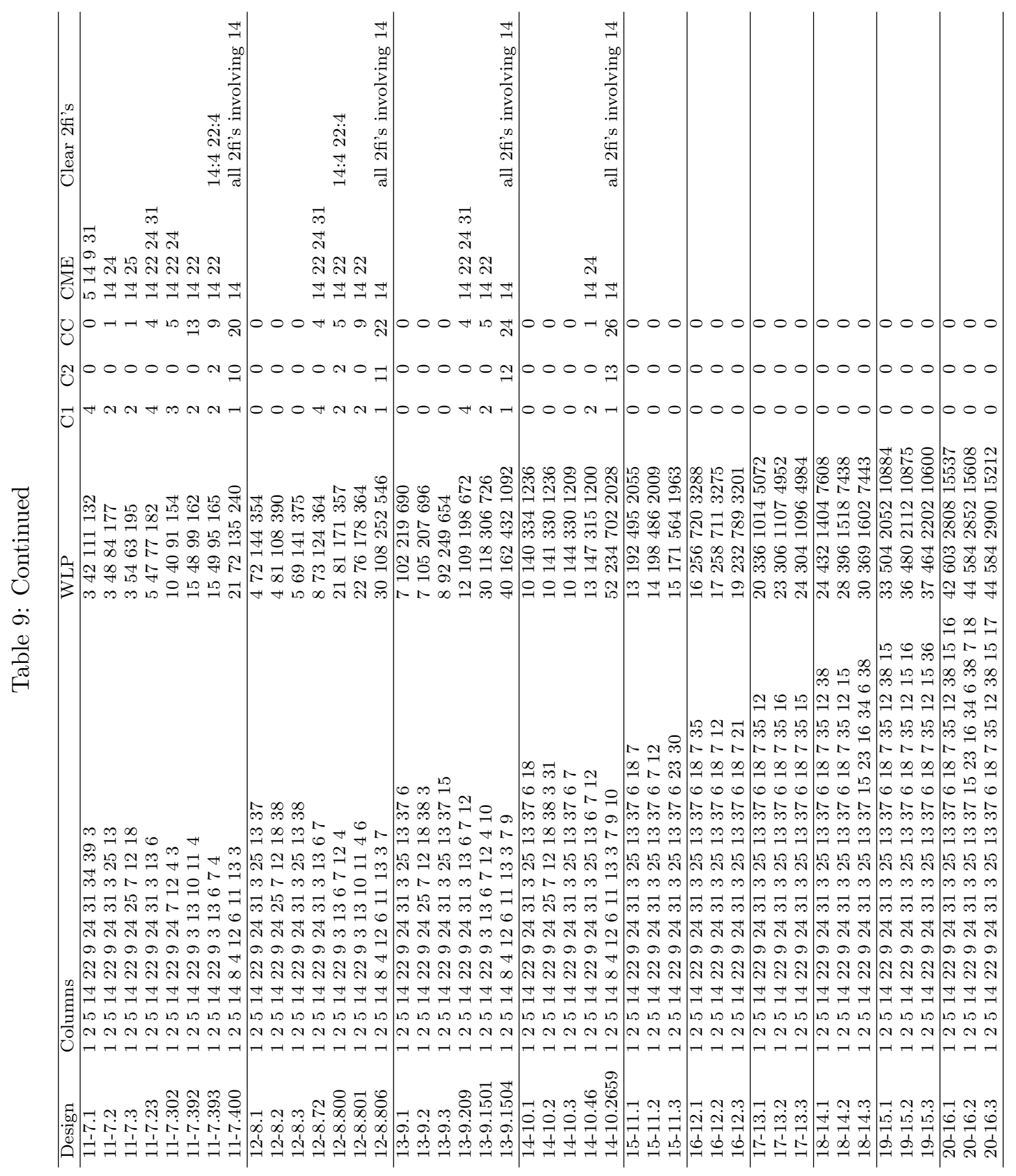




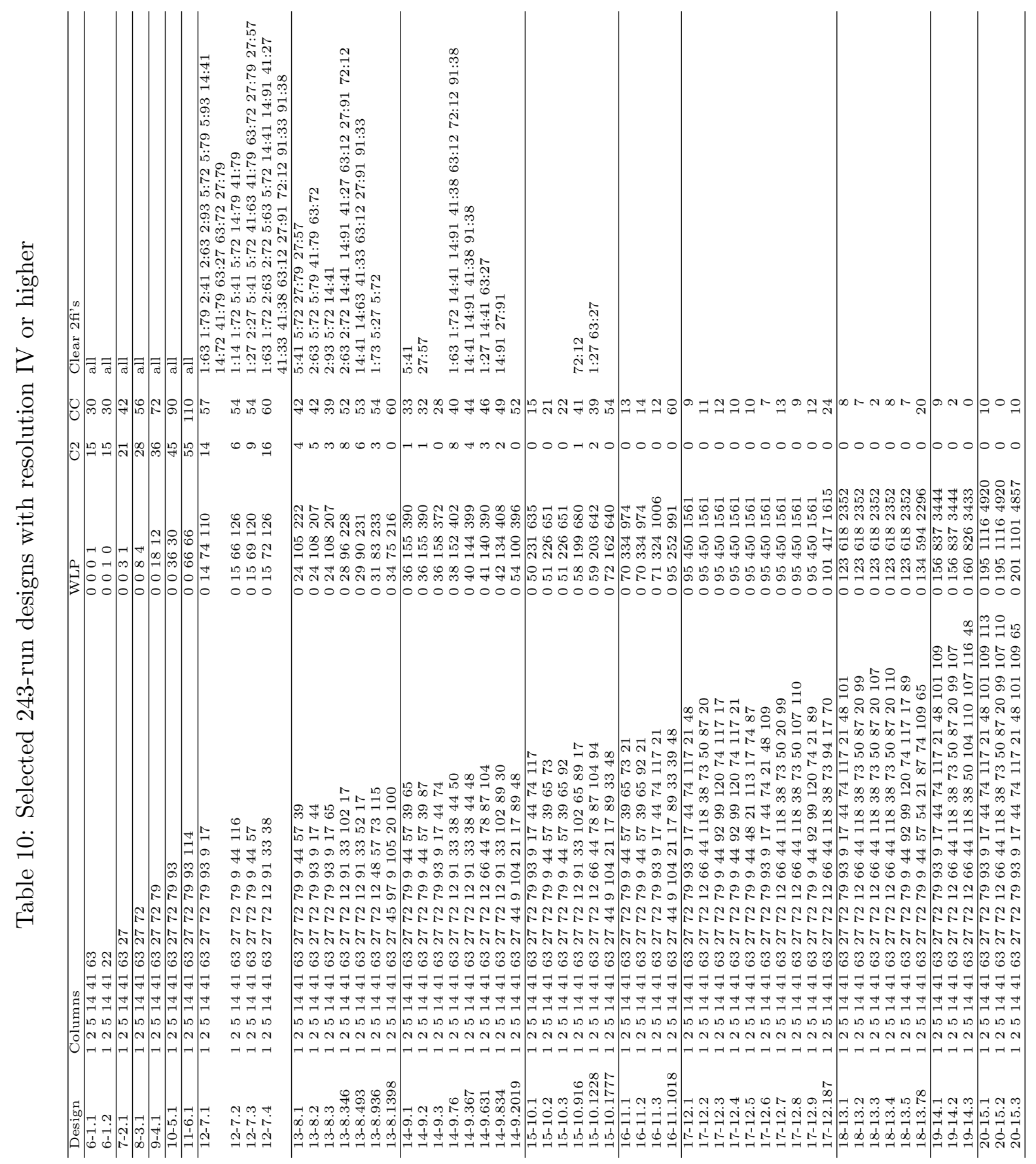


Table 11: Selected 729-run designs with resolution V or higher

\begin{tabular}{|c|c|c|}
\hline Design & Columns & WLP \\
\hline $7-1.1$ & 1251441122185 & 00000 \\
\hline $7-1.2$ & 125144112263 & 00001 \\
\hline $7-1.3$ & 125144112222 & 0010 \\
\hline $8-2.1$ & $12251441 \quad 12263149$ & 00004 \\
\hline $8-2.2$ & 125144112218527 & 0012 \\
\hline $8-2.3$ & 125144112218523 & 0020 \\
\hline $9-3.1$ & 1255144112263149201 & 000012 \\
\hline 9-3.2 & 125144112263149166 & 0027 \\
\hline $9-3.3$ & 125144112218527206 & 0034 \\
\hline $10-4.1$ & 125144112263149201236 & 00030 \\
\hline $10-4.2$ & 12514411226314920136 & 00517 \\
\hline $10-4.3$ & 125144112263149166188 & 00614 \\
\hline $11-5.1$ & 125144112263149201236315 & 000066 \\
\hline $11-5.2$ & 12514411226314920123636 & 00939 \\
\hline $11-5.3$ & 1251441122631492013654 & 001233 \\
\hline $12-6.1$ & 1255144112263149201236315336 & 0000132 \\
\hline $12-6.2$ & 12514411226314920123631536 & 001581 \\
\hline $12-6.3$ & 12514411226314920123636105 & 002166 \\
\hline $13-7.1$ & 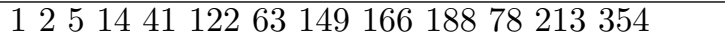 & 003991 \\
\hline $13-7.2$ & 12514411226314920123636173115 & 004486 \\
\hline $13-7.3$ & 12514411226314916618854242105 & 004580 \\
\hline $14-8.1$ & 125514411226314916618854242105212 & 0070140 \\
\hline
\end{tabular}

Note: All main effects and 2fi's are clear. 